\title{
Review
}

\section{Colorants: Their Interaction with Flavor in Product Development}

\author{
Deepti Dabas, PhD* \\ Lead Scientist and Senior Manager, Kalsec ${ }^{\circledR}$ Inc., 37 I 3 West Main Street, Kalamazoo 49006, Michigan, USA
}

\section{"Corresponding author}

Deepti Dabas, $\mathrm{PhD}$

Lead Scientist and Senior Manager, Kalsec ${ }^{\circledR}$ Inc., 37 I 3 West Main Street, Kalamazoo 49006, Michigan, USA; E-mail: dabasd@gmail.com

\author{
Article information

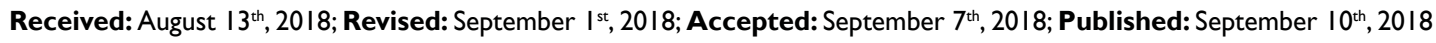

\section{Cite this article}

Dabas D. Colorants:Their interaction with flavor in product development. Adv Food Technol Nutr Sci Open J. 2018; 4(I): 17-22. doi: 10.17। 40/AFTNSOJ-4-I48

\section{| ABSTRACT |}

Color is an important part of product appeal and is a critical criterion in a consumers' ultimate selection of foods and beverages. The color of a food or beverage not only acts as an indicator of quality and freshness, it can also elicit expectations of flavor. The utilizationof naturally sourced colors in food and beverages is increasing at a faster pace compared to synthetic colors. Naturally occurring molecules having a higher molar extinction are favored as colorants due to higher efficiency of use in applications compared to other naturally existing molecules. Flavor is another critical sensory attribute in food and beverages and therefore it is important to study and understand the interaction of color and flavor while developing products. Color needs to be dispersible with the flavor as well as in the application. Ideally color should not alter the flavor profile of the food or beverage, nor should it negatively impact the stability of the flavor system. Ultimately, colors that align well with the flavor and with the application should be selected. Colorant extracted from radish is an example of a color system which imparts flavor and therefore may need special processing. However, different radish sources may have different flavor thresholds. One final consideration when choosing a natural colorant for development into a food or beverage product is that different consumers may have varying degrees of sensitivities to the flavor of these color products.
\end{abstract}

\section{Keywords}

Natural colorant; Color flavor interaction; Beverages; Fruit preparations; Colors imparting flavor.

\section{INTRODUCTION |}

$\mathrm{T}$ he purpose of this review is to discuss critical aspects and considerations of color/flavor interaction during the development of food and beverage products. This review covers the science of why we see color, why colors are added to food and beverage, and provides a definition of naturally sourced colorants. In addition, color/flavor interaction is described through beverage and fruit preparation applications. Discussions regarding dispersibility, flavor impact and stability of colorants are also included.

\section{COLOR AND VISIBLE LIGHT |}

Color is the effect on the eye caused by light waves in the visible region. The visible region ranges from $380 \mathrm{~nm}$, higher energy/ shorter wavelength violet light, to $700 \mathrm{~nm}$, lower energy/longer wavelength red light. The speed of light being a constant, c, and knowing the speed of light is equal to the product of the frequency $(\nu)$ and wavelength of light $(\lambda), c=\nu \lambda$, red light has a lower frequency wave than violet, which has the highest frequency of light wave in the visible region. Figure $1^{1}$ highlights the visible region of the electromagnetic spectrum. It is of interest to note 
that color can be expressed quantitatively by either frequency or wavelength. A dispersive prism is an excellent tool to separate and visualize the color components of white light. White light waves are refracted within the prism, shorter wavelengths of light are refracted more than longer wavelengths, thus separating the light into spectral colors.

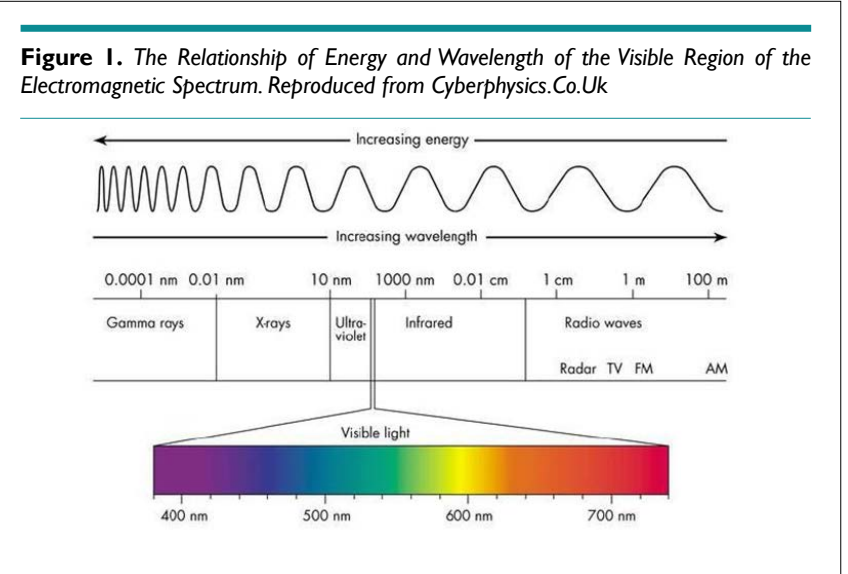

\section{Why Do We See Different Objects as Different Colors?}

When white light strikes an object, visible light is either absorbed or reflected depending upon the chemical structure of the molecules present in the object. If all visible light is absorbed the object is perceived as black. If all visible light is reflected from the object the object is perceived as white. The appearance of color, say red, occurs when a red light is reflected, and all other colors of the visible region are absorbed by an object, or less frequently, when the complimentary color of red, green, is absorbed and all other colors are reflected by an object. Figure 2 illustrates why we see black, red and white colored objects.

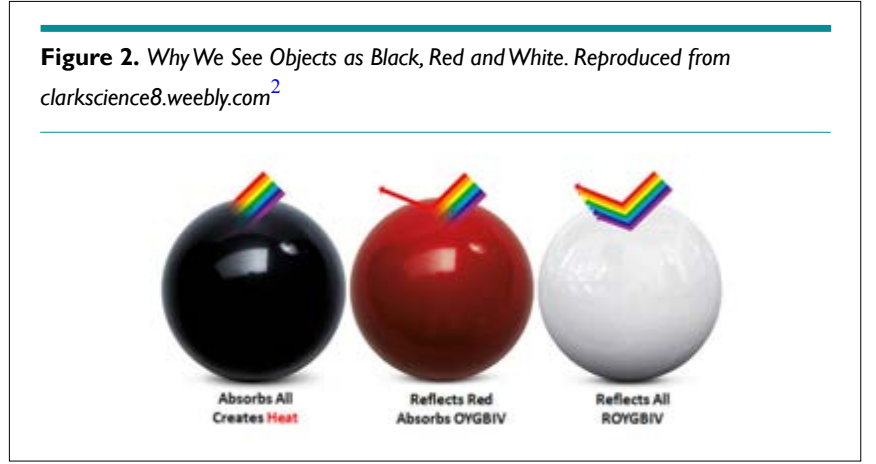

Aromatic compounds having hetro elements (elements other than carbon and hydrogen) in their structure and those possessing conjugated bonds are typically the best candidates to be colorants. These compounds selectively absorb and reflect wavelengths of visible light to produce their unique colors as described above. Such absorption is associated with energy changes within the molecule. This energy difference between the ground and ex- cited state is proportional to the observed frequency (inversely proportional to wavelength) of absorbed light. When light strikes a compound, the light's energy is used to promote electrons from bonding or nonbonding orbitals to empty anti-bonding orbitals. Each electron transfer utilizes energy from light and bigger jumps $\left(\sigma^{*}\right.$, sigma anti-bonding orbital) need more energy than smaller jumps $\left(\pi^{*}\right.$, pi antibonding orbital) - sigma antibonding orbital lies above the pi antibonding orbital in terms of energy. The higher the energy jump, the greater absorption of light at a higher frequency is needed-hence greater the frequency, greater is the energy. Or the larger the energy jump, the lower wavelength of the light absorbed- hence molecules displaying this phenomenon will appear orange or red. As the resonance or resulting delocalization in a molecule increases, it is easier for the electrons to be excited and hence more likelihood of the molecule being colored. For example, beta-carotene has much more delocalization with 11 carbon conjugated double bonds. Thus, it is much more intensely colored than retinol which has only 5 conjugated bonds and is light yellow colored. Hence beta-carotene has a higher molar extinction coefficient (a measure of how strongly a compound absorbs radiation) than retinol.

\section{WHY IS COLOR ADDED?}

Color is added to many processed foods and beverages and is an important part of product appeal. Color is the most notable attribute in food evaluation making it to be a critical component of intent to purchase. Appearance and color are the visual elements to which our eyes, minds, emotions and palates are sensitive. The following sections detail additional reasons as to why color plays such an important role in foods and beverages.

\section{Color Acts as Indicator of Quality and Deterioration for Fruits and Vegetables}

Naturally-derived colorants are pigments extracted from fruits and vegetables, many of which change as a plant proceeds through maturation and ripening. Inherent pigments imparting color quality are fat-soluble chlorophylls (green), carotenoids (yellow, orange and red), water-soluble anthocyanins (red, blue), flavonoids (yellow) and betalains (red). In addition, enzymatic and non-enzymatic browning reactions may result in the formation of brown, grey and black colored pigments. The enzymes involved in browning reactions include polyphenol oxidase, which catalyzes the oxidation of polyphenolic compounds such as in apple juice. ${ }^{3}$ The quality deterioration as responding to various stresses during storage can be evaluated in terms of changes in color (green -chlorophyll, yellow and orange-carotenoids, brown-enzymatic browning). Other parameters like texture (stiffness, firmness and consistency), off flavor soapy-like, stearin-like and paraffin-like, nutrition (antioxidant, polyphenol and ascorbic acid), composition (starch, sugar) and sensory (flavor, taste, color, consistency, appearance and overall attributes) are also affected. ${ }^{4}$ An example where color is indicative of deterioration of product quality is a banana whose peel has blackened. 


\section{Foods and Beverages Induce Pre-Consumption Expectations about Flavor Identity}

Color intensity is a cue for the quality of foods as well as the intensity of taste. Food color also elicits expectations of nutritional value with red colored foods perceived as higher energy density than green colored foods. ${ }^{5}$ We associate colors with certain flavors-such as yellow color is generally associated with lemon flavor and red color with berry flavors (raspberry, blueberry and strawberry). Color, therefore, elicits expectations of certain flavors and guides us towards identifying those flavors.

\section{Color Variety Impacts not Only the Perception of Taste but also the Amount Consumed}

As an example, studies have shown that multicolored candy is consumed in higher amounts than single colored candy. This phenomenon has also been observed in other food groups, including fruits and vegetables. In one research study the perceived color variety of the meals and its effects on consumption of different food groups (fruits, starches, protein, vegetables, sugar, dairy) was evaluated. The increase in perceived meal variety was associated with an increased proportion of vegetables consumed and reduced the proportion of extra sugary content consumed. ${ }^{5}$

\section{NATURAL COLORS}

The Food and Drug Administration (FDA) has not officially defined 'natural colors', however, it is generally understood and an industry standard that natural colorants are defined as pigments extracted and isolated from natural sources. Examples of natural colors are paprika oleoresin extracted from paprika pods, curcumin derived from turmeric rhizome and chlorophyll derived from alfalfa. The polarity of the natural pigment determines the solvent and solvent ratio in the extraction. Residual solvent limits in the final natural colorant product are set by FDA and are in the ppm range. Artificial colors on the other hand are often manufactured from non-sustainable synthetic materials such as coal tar or petroleum derivatives but are generally more stable and of lower cost than natural colors. The food and beverage color market is projected to grow at a compound annual growth rate (CAGR) of $8.40 \%$ and reach USD 3.75 billion by 2022 ; natural colorants are expected to have a $53.6 \%$ share of this market (USD 2.01 billion). This rate of growth is higher than that of synthetic colors. The consumer demand for 'clean'labelled productshas resulted in the projection of a higher rate of growth of natural colorants over synthetics, ${ }^{6}$ with the largest market of natural colorants expected to be in North America.

\section{CONSIDERATIONS IN MAKING A PRODUCT CONTAINING BOTH COLOR AND FLAVOR}

For the purpose of this review, naturally sourced colors and flavors will be discussed. Pigments generally contain higher molecular weight molecules as compared to flavor molecules. Substances responsible for odor perception are volatile (high vapor pressure) and are generally low molecular weight compounds. The struc- tural and property differences between natural colors and flavors are important to know and understand when adding to food and beverage applications.

\section{Dispersibility of Color and Flavor}

Due to the difference in their physical and chemical properties, color and flavor may not disperse together. This may call for adding specific additives to disperse them uniformly in the application. Different emulsifiers are commonly used to disperse color or flavor or both into the matrix such as lecithin or polysorbates. The use of a specific emulsifier and its concentration is determined by the properties of the matrix. For example, to attain a strawberry flavor in a beverage application, emulsifiers would be needed to disperse the flavor constituents which are comprised of particular esters, terpenes, and furans; ${ }^{7}$ however, a black carrot based colorant may work for a beverage application and will not require any emulsifier for dispersion since it is water soluble.

\section{Color Imparting Flavor}

When developing products with both colors and flavors considerations need to be madeaccording to the application. A common concern for scientists developing new products is the odor/flavor system associated with the concentrated color raw material. Most color raw materials are developed with high concentrations to allow use in multiple applications and to save on transportation costs. Because of the high potency of the commercial colorants they may possess noticeable odor and flavor in their concentrated form. However, when the colorant is dosed into the application, it may not cause any flavor impact. Therefore, the formulator should test the product in his desired application before looking for color alternatives.

\section{Color Affecting the Stability of Flavor}

Keeping color and flavor together in a food matrix can have its challenges. For example, the stability of one may be affected by the other. Some color pigments may undergo rancidity such as carotenoids. Carotenoids may get oxidized, generate off flavors and lose color; these oxidized products then can cause off flavor in an application. However, carotenoids may also enhance original flavor stability by protecting other constituents including flavor.

\section{Selecting Suitable Color for a Flavor}

Lemon flavor is generally associated with yellow color; lemon gets its flavor profile from molecules like citral, geranial, lemonal etc. ${ }^{3}$ Colors are then selected based on this association and different yellow colors can be used such as orange carrot extract, turmeric oleoresin, gardenia yellow or safflower. It is crucial to know the properties of these pigments and select the one that aligns with that of flavor product. Factors such as water solubility, light stability and local labeling regulations should be considered. For example, sensitivity of turmeric to light impacts its use in certain application like beverages, while it is quite stable in a hard candy. 


\section{Regulatory Landscape}

Regional regulations can dictate what color and flavor systems may be utilized in formulating a food or beverage. Most flavors and excipient ingredients in food and beverage products have traversed the generally recognized as safe (GRAS) affirmation route, but color additives are specifically prohibited from being determined GRAS outside of the FDA petition process. Therefore, all food colorings must go through a pre-market review and approval by the FDA to be included on the approved colorant listing. A food ingredient found to be GRAS for one technological function, e.g., as an antioxidant or acidulant, might also be the subject of a color additive petition if it also imparts color into an application. In addition, if an approved or exempt colorant is discovered through research and testing to have an additional function in a food or beverage, e.g., an antioxidant or an acidulant, the colorant may be to subject to an external GRAS affirmation as a food ingredient for this non-color function. ${ }^{8}$ It is important to remember that regulatory definition of natural varies for colors and flavors. For colors, the FDA has a regulatory definition that if an ingredient is derived, it may not be called natural universally (example, gardenia blue) and for derived flavors there is a specific definition that allows them to be categorized as natural if they fit the outlined requirements and use approved ingredients (example, maillard reaction products). In Europe, ingredients added as colorants are classified either as color additive ( $\mathrm{E}$ numbers), or as coloring foods. E numbers are used for additives used to color foods; recently coloring foods have come into the forefront and regulations around them are still being developed and adopted. Although there are no regulations inhibiting the use of artificial colorants, studies like Southampton have discouraged the use of artificial colorants in Europe. ${ }^{9}$

\section{SPECIFIC APPLICATIONS CONTAINING COLOR AND FLAVOR}

\section{Beverages}

Beverages can be challenging applications as they may require color and flavor added in the form of an emulsion that may be exposed to light, high water activity and other environmental factors. Beverages can be used in different forms such as 'ready to drink' or more concentrated forms where the consumer adds water at 6-8 times the concentration level. Some pigments, such as turmeric, may not remain stable over time in this relatively harsh environment. Colors and flavors may also be formulated with different emulsifiers which can affect performance. For example, protein based emulsifiers and pectin emulsifiers may affect the flavor and color respectively and after addition may precipitate at an approximate $\mathrm{pH}$ of $3 .{ }^{10}$ This could cause the beverage to coalesce due to oppositely charged particles of these emulsifiers and would mean separation of color and flavor even though flavor precipitation may not be visible.

Generally, flavor oils have much lower density in the aqueous phase which may be a factor causing emulsion breakdown. To prevent this, weighting agents may need to be added to create a stable emulsion system. Flavor consists of not only the flavor molecule but are generally mixtures of flavor oil and non-flavor oils. As an example, citrus-based flavor oils such as orange, lemon, lime and grapefruit fall into this category. These oils are highly complex substances that contain a variety of different kinds of molecules with different structures, polarity and chemical properties. The primary constituents are the hydrocarbon terpenes (monoterpene and sesquiterpenes) like myrcene, limonene, citral, geranial etc., but they also contain other compounds responsible for flavor such as alcohols, ketones, acids and esters. ${ }^{11}$ These compounds need to be incorporated into an emulsion as they have limited water solubility. Infact, these latter group of compounds have some water solubility which may lead to Ostwald ripening. Ostwald ripening is the growth of bigger globules at the expense of smaller ones in an emulsion system; if left unchecked it may ultimately lead to creaming and breakdown of the emulsion. Adding different emulsions (flavor and color) into a beverage may also cause creaming based on different particle sizes. ${ }^{12}$. Thus, it is important to keep the particle sizes of both the color and flavor emulsion in a similar range.

For some products, color and flavor may be present in separate phases unlike the oil phase examples discussed above. A raspberry flavored beverage, for example, where the flavor oil may be in the oil phase and the color (typically an anthocyanin) will be in a continuous phase, is less challenging. Anthocyanins can be radish, cabbage, black carrot based-sometimes berry anthocyanins are also used which may not be as stable as vegetable based sources. In most cases, the beverages do need to be pasteurized after adding the colorants-in such cases stability studies with the actual beverage matrix should be conducted before choosing the source and concentration of the color. Vegetable based anthocyanins have more likelihood of surviving the pasteurization conditions; black carrot is a good example.

\section{Fruit Preparations}

Fruit preparations are used in many applications such as yogurt, baking and ice-cream where they contribute the color, flavors and texture of the fruit. This application does require water dispersibility and oil soluble colors and flavors may need to be dispersed or emulsified before adding to a fruit preparation. Many similar considerations with respect to emulsifiers apply as referenced in beverage applications. Fruit preparation processing should be done in a vacuum kettle to retain flavor volatiles by processing at lower temperatures to a predetermined level of solids which is typically $65 \% .^{10}$ At a higher brix level, boiling temperature is generally 5-7 times higher than the boiling pressure of water. This can lead to color loss, so this should be a consideration when colors are added. To avoid this, color should be added at the end of the manufacturing process when possible. It is important to note that the nonvolatile portion of flavor may also degrade on heating.

\section{COLORS BRINGING THEIR FLAVOR TO PRODUCTS}

In some cases, unwanted flavors may accompany colorants. Some color pigments are known for their unwelcoming flavor such as 
beet and radish pigments. Removal of flavor may require further processing after color extraction. In the case of radish extract, hydrogen disulfide, dimethyl disulfide and other sulfur-based compounds are responsible for this odor and flavor. Radish is known for its off flavor which is derived from glucosinolates. ${ }^{13}$ As the vegetable is crushed and prepared for extraction, myrosinase (an enzyme) acts on the vegetable and creates different molecules responsible for the characteristic radish flavor and additional off flavors.

$\mathrm{Kalsec}^{\circledR}$ conducted a study on the interaction between flavor and odor in radish pigments. Determination of odor and taste threshold by forced choice ascending concentrations series method of limits was used. Panelists evaluated the products in order of increasing color strength and were asked to complete an entire series of seven stations, increasing in concentrations. Best estimate threshold (BET) was determined and the following observations were reported:

- Each of these two samples had different best estimate thresholds which was expected. The mean of sample 1 was found to be $230 \mathrm{ppm}$ whereas the mean for sample 2 was estimated at 544 ppm. This reflects the challenge of formulating.

- The individual BET for each sample varied immensely among participants. For selected panelists, the range was 39.4 to 1623.5 ppm. Thus, some panelists are very sensitive to these compounds while others may not be able to perceive these compounds.

This study shows that while switching supplies of a naturally sourced colors, the possible impact on flavor must be considered.

\section{CONCLUSION}

Color is an important sensory attribute in foods and beverages and can play a key role in a consumers' selection process to purchase a product. There are many reasons colors are added to foods and beverages. Not only are colorants a crucial attribute while selecting food for consumption, they are associated with the quality attributes of the foods and beverages. Colors are also associated with certain flavors and when added, point our senses towards that flavor. For example, red color added to a strawberry flavored candy. Naturally sourced colors are becoming more significant as the demand for fewer artificial ingredients increases. Formulating with naturally sourced colors and flavors can be challenging as they may have very different chemical properties. Also, other ingredients like emulsifiers or diluents are usually added to these colors and flavors and can interfere with the stability of finished product. Therefore, it is important to know the interaction and understand the stability of these compounds before introducing a new product to market. Some colors can bring in their own flavor and this should be evaluated for its impact on the application. This can impact the recommendation of naturally sourced colors chosen for a particular application. As observed in the sensory study, two sources of radish had different BET values and indicates the different concentration of active odor molecules in the two sources. This indicates a thorough comparison of color and its flavor is important when evaluating color sources. Natural sources are expected to have some variation regarding the ratio of different color and flavor components. Food application formulators should consider these aspects when formulating with naturally sourced colors. Liquid color extracts and oleoresins can help to provide reduced variation in formulating.

\section{ACKNOWLEDGEMENT}

The author acknowledges the help from Robin Boyle for carrying out the sensory study. The author is thankful to Eric Engelman and Gary Augustine for reviewing this paper.

\section{CONFLICTS OF INTEREST}

The author declares no conflict of interest.

\section{REFERENCES}

1. Cyberphysics. The Electromagnetic Spectrum: The family of Light. Website: http://www.cyberphysics.co.uk/topics/light/emspect.htm. Accessed August 12, 2018.

2. Clark. How we see in color. Website: http://clarkscience8.weebly.com/how-we-see-in-color.html. Accessed August 12, 2018.

3. Barrett DM, Beaulieu JC, Shewfelt R. Color, flavor, texture, and nutritional quality of fresh-cut fruits and vegetables: Desirable levels, instrumental and sensory measurement, and the effects of processing. Crit Rev Food Sci Nutr. 2010; 50(5): 369-389. doi: 10.1080/10408391003626322

4. Waraporn Boonsupthip DRH, Jinnipar Choachamnan. Quality monitoring and management during storage and distribution of frozen vegetables. In: Hui YH, Evranuz EÖ. Ed, Handbook of Vegetable Preservation and Processing, Second Edition. Boca Raton, USA: CRC Press. 2015; p 28.

5. König LM, Renner B, Colourful=healthy? Exploring meal colour variety and its relation to food consumption. Food Quality and Preference. 2018; 64: 66-71. doi: 10.1016/j.foodqual.2017.10.011

6. Markets and markets. Food Colors Market: Global Forecast to 2022. USA: Market Publishers; 2016.

7. Schwieterman ML, Colquhoun TA, Jaworski EA, et al. Strawberry flavor: Diverse chemical compositions, a seasonal influence, and effects on sensory perception. PLoS One. 2014; 9(2): e88446. doi: 10.1371/journal.pone.0088446

8. Griffith JC. Coloring foods and beverages. Food Technology. 2005; 59: 38-44.

9. Scott-Thomas C. EFSA calls for safety tests for six food dyesincluding five southampton colours; 2013. 
10. Kendrick A. 7 - Coloring aqueous food types. In: Handbook on Natural Pigments in Food and Beverages. Cambridge, UK: Woodhead Publishing. 2016; 163-177.

11. Hausch BJ, Lorjaroenphon Y, Cadwallader KR. Flavor chemistry of lemon-lime carbonated beverages. J Agric Food Chem. 2015; 63; 112-119. doi: 10.1021/jf504852z

12. McClements DJ, Henson L, Popplewell LM, Decker EA, Choi
SJ. Inhibition of Ostwald ripening in model beverage emulsions by addition of poorly water soluble triglyceride oils. J Food Sci. 2012 ;77(1): C33-38. doi: 10.1111/j.1750-3841.2011.02484.x

13. Chen W, Karangwa E, Yu J, et al. Characterizing red radish pigment off-odor and aroma-active compounds by sensory evaluation, gas chromatography-mass spectrometry/olfactometry and partial least square regression. Food Bioprocess Technol. 2017; 10: 1337-1353. doi: 10.1007/s11947-017-1904-5 